\title{
Influence of chitosan coating on protein-based nanohydrogels properties and in vitro gastric digestibility
}

\author{
Ana I. Bourbon*, Ana C. Pinheiro, Miguel A. Cerqueira, António A. Vicente \\ CEB, Centre of Biological Engineering, University of Minho, Campus de Gualtar, 4710-057 Braga, Portugal
}

\section{A R T I C L E I N F O}

\section{Article history:}

Received 11 November 2015

Received in revised form

22 January 2016

Accepted 1 March 2016

Available online 8 March 2016

\section{Keywords:}

Protein hydrolysis in gastric conditions

Controlled release

Caffeine

\begin{abstract}
A B S T R A C T
Chitosan coating was applied in Lactoferrin (Lf)-Glycomacropeptide (GMP) nanohydrogels by layer-bylayer coating process. A volume ratio of 10 of Lf-GMP nanohydrogels $\left(0.2 \mathrm{mg} \mathrm{mL}^{-1}\right.$, at $\left.\mathrm{pH} 5.0\right)$ to chitosan (1 mg mL $\mathrm{mL}^{-1}$, at $\mathrm{pH} 3$ ) demonstrated to be the optimal condition to obtain stable nanohydrogels with size of $230 \pm 12 \mathrm{~nm}$, a PdI of $0.22 \pm 0.02$ and a $\zeta$-potential of $30.0 \pm 0.15 \mathrm{mV}$. Transmission electron microscopy (TEM) images showed that the application of chitosan coating in Lf-GMP did not affect the spherical shape of nanohydrogels and confirmed the low aggregation of nanohydrogels in solution. The analysis of chemical interactions between chitosan and Lf-GMP nanohydrogels were performed by Fourier transform infrared spectroscopy (FTIR) and by circular dichroism (CD) that revealed that a specific chemical interaction occurring between functional groups of protein-based nanohydrogels and active groups of the chitosan was established. The effect of chitosan coating on release mechanisms of LfGMP nanohydrogels at acid conditions $\left(\mathrm{pH} 2,37^{\circ} \mathrm{C}\right)$ was evaluated by the encapsulation of a model compound (caffeine) in these systems. Linear Superposition Model was used to fit the experimental data and revealed that Fick and relaxation mechanisms are involved in caffeine release. It was also observed that the Fick contribution increase with the application of chitosan coating. In vitro gastric digestion was performed with Lf-GMP nanohydrogels and Lf-GMP nanohydrogels with chitosan coating and it was observed that the presence of chitosan improve the stability of Lf and GMP (proteins were hydrolysed at a slower rate and were present in solution by longer time). Native electrophoreses revealed that the nanohydrogels without coating remained intact in solution until $15 \mathrm{~min}$ and with chitosan coating remained intact until $60 \mathrm{~min}$, during gastric digestion.
\end{abstract}

(C) 2016 Elsevier Ltd. All rights reserved.

\section{Introduction}

Protection of active compounds and the ability to maintain them active until release near the target cell tissue, is a great challenge for the food and pharmaceutical industries. Nanostructures are considered promising systems due their small dimensions that enables versatile advantages for targeted, site-specific delivery purposes as long as they can penetrate circulating systems and reach specific sites in the body at a suitable time (Cerqueira et al., 2014; Martins et al., 2015).

Protein nanohydrogels are considered an attractive vehicle to encapsulate and delivery different bioactive compounds, due their large network, low toxicity, high biodegradability, biocompatibility and ability to deliver bioactive compounds into and/or across the

\footnotetext{
* Corresponding author.

E-mail address: isabelbourbon@gmail.com (A.I. Bourbon).
}

Gastro Intestinal (GI) mucosa (Somchue, Sermsri, Shiowatana, \& Siripinyanond, 2009). Depending on the nature of the bioactive compounds incorporated in nanohydrogels it is possible to observe different release mechanisms during the digestion process. Hydrophilic compounds can be released from a protein matrix by diffusion, whereas lipophilic compounds are released mainly by enzymatic degradation of the protein matrix in the GI tract (Wang, Tian, \& Chen, 2011). The degradation of protein matrix in GI tract is an obstacle to the delivery of the encapsulated compound at specific target (e.g. mouth, stomach, small intestine or colon). Nanostructures composed by proteins or peptides demonstrated to have a high level of GI degradation by digestive enzymes (Donato-Capel et al., 2014; Shaji \& Patole, 2008).

Gastric conditions are determinant in digestion of protein structures. The stomach ensures the denaturation of proteins by the gastric acidity and also the proteins hydrolyse by pepsin (Yvon, Beucher, Scanff, Thirouin, \& Pelissier, 1992). Nabil, Gauthier, Drouin, Poubelle, and Pouliot (2011) observed that almost of 
bovine whey protein extract (BWPE) was hydrolysed in gastric compartment and no intact whey protein was detected in intestinal compartments (Nabil et al., 2011). Moreover, these authors observed that after $1 \mathrm{~h}$ of gastric digestion $61 \%$ of BWPE was already hydrolysed. The degradation of proteins compromises the delivery of these active compounds in intestine were occurs the absorption. One of the strategies used to improve the stability of nanostructures in gastro conditions, is the application of a coating to prevent the hydrolysis of proteins by proteolytic enzymes (Somchue et al., 2009). Layer-by-Layer (LbL) deposition technique is one of the methods used in different templates (from hard and planar to rigid particles, and more recently in soft and porous templates such as nanohydrogels) to improve the stability, functional and mechanical properties of different structures (Boddohi, Almodóvar, Zhang, Johnson, \& Kipper, 2010; Hirsjärvi, Qiao, Royere, Bibette, \& Benoit, 2010; Kittitheeranun et al.; Kotov, 2003; Sato, Yoshida, Takahashi, \& Anzai, 2011; Wong, Müller, Diez-Pascual, \& Richtering, 2009). LbL assembly is based on the electrostatic interaction between oppositely charged polyelectrolytes alternatively adsorbed onto an appropriate template (Decher, 2003).

Chitosan, is a cationic polysaccharide obtained by deacetylation of chitin, which is the major constituent of exoskeleton of crustaceous animals. Chitosan is nontoxic, biodegradable, and biocompatible (Khopade \& Caruso, 2004; Li, Wang \& Wu, 1998). This polysaccharide is used to enhance bioactive compounds absorption in epithelium and its ability to protects structures in gastric environmental has been reported (Chew, Tan, Long, \& Nyam, 2015; Rastall, 2010). In order to control the degradability of protein nanohydrogels and increase the residence time of proteins in the gastric conditions, chitosan coating has been applied in nanohydrogels composed by lactoferrin (Lf) and glycomacropeptide (GMP).

This study was carried out to evaluate the ability of chitosan coating to affect Lf-GMP nanohydrogels properties and their stability during gastric digestion. The influence of chitosan coating on Lf-GMP nanohydrogels was also evaluated in controlled release properties of caffeine. This study shows a successful attempt to use protein-based systems in combination with chitosan to allow protection and delivery of bioactive compounds to specific targets (e.g. intestinal epithelium) for bioavailability improvement.

\section{Materials and methods}

\subsection{Materials}

Purified Lf powder was obtained from DMV International (USA) and it is composed by $96 \%$ protein, $0.5 \%$ ash, 3.5\% moisture and the an iron content is around $120 \mathrm{ppm}$ (composition expressed as a dry weigh percentage). Commercial GMP was obtained from Davisco Food International, INC. (Le Sueur, USA) and its reported composition is: $82.5 \%$ protein, $1 \%$ fat, $7 \%$ ash and $7 \%$ moisture. Chitosan of low molecular weight (molecular weight ranging between 50 and $100 \mathrm{KDa}$ and with a deacetylation degree $\geq 95 \%$ ) was purchased from Sigma-Aldrich (St. Louis, MO, USA). Lactic acid (90\%) was obtained from Acros Organics (Geel, Belgium). Caffeine was purchased from AnalaR NORMAPUR ${ }^{\circledR}$ (Ireland) and Amicon ${ }^{\circledR}$ Ultra-0.5 with a molecular cut-off $3 \mathrm{kDa}$ centrifugal filter devices were purchased from Millipore Corp., Ireland. All the samples were dissolved in deionized water purified to a resistance of $15 \mathrm{M} \Omega$ (Millipore, France).

Cellu-Sep H1, dialysis membrane was obtained by Membrane filtration products, USA. To adjust the solutions $\mathrm{pH}$ it was used hydrochloric acid, purchased from Panreac, Spain.
For the simulated gastric juice, it was used pepsin from porcine gastric mucosa $\left(600 \mathrm{U} \mathrm{mL}^{-1}\right)$, lipase from porcine pancreas $\left(40 \mathrm{U} \mathrm{mL}^{-1}\right)$ and different salts $\left(\mathrm{NaCl}, \mathrm{KCl}, \mathrm{CaCl}_{2}\right.$ and $\left.\mathrm{NaHCO}_{3}\right)$ to prepare the gastric electrolyte solutions; all of them purchased from Sigma (St. Louis, USA). All other chemicals used in this study were reagent grade.

\subsection{Preparation of Lactoferrin-Glycomacropeptide nanohydrogels}

Lf-GMP nanohydrogels were prepared as described in Bourbon et al. (2015). Briefly, 1.25 $\mu \mathrm{M}$ of Lf and 8.3 $\mu \mathrm{M}$ of GMP were dissolved separately, in deionized water purified at $25^{\circ} \mathrm{C}$. The $\mathrm{pH}$ values of biopolymer solutions were separately adjusted to 5.0 , with $0.1 \mathrm{~mol} \mathrm{~L}^{-1}$ of hydrochloric acid. Lf aqueous solution was added dropwise into GMP aqueous solution with gently stirring until final molar ratio (MR) 1:7 of Lf to GMP. The Lf-GMP mixture solution was heated in a closed bath, at $80{ }^{\circ} \mathrm{C}$ during $20 \mathrm{~min}$.

\subsection{Preparation of chitosan coating on Lf-GMP nanohydrogels}

The chitosan was assembled on the Lf-GMP nanohydrogels by LbL deposition technique.

After Lf-GMP nanohydrogels production process, the nanohydrogels were added to a chitosan solution $\left(1 \mathrm{mg} \mathrm{mL}^{-1}, \mathrm{pH} 3\right.$, dissolved in $1 \%$ of lactic acid) at different volume ratios (VR) of LfGMP nanohydrogels to chitosan, with constant stirring of $200 \mathrm{rpm}$ during $15 \mathrm{~min}$, creating the nanohydrogels with a coating.

\subsection{Characterization of Lf-GMP nanohydrogels coated with chitosan}

\subsection{1. $\zeta$-potential measurements}

The $\zeta$-potential of coated Lf-GMP nanohydrogels was determined by dynamic light scattering (DLS; Zetasizer Nano ZS, Malvern Instruments, UK). Each sample was analysed in a folded capillary cell. The $\zeta$-potential values are the average of nine successive measurements.

\subsubsection{Size}

Nanohydrogels with chitosan coating were characterized in terms of size distribution (by number) and polydispersity index (PdI) using a Dynamic Light Scattering (DLS) apparatus (Zetasizer Nano ZS, Malvern Instruments, UK) equipped with a He-Ne laser at a wavelength of $633 \mathrm{~nm}$. All measurements were performed at $25{ }^{\circ} \mathrm{C}$. Each measurement of size and PdI was performed with a detection angle of $173^{\circ}$. The results are given as the average \pm standard deviation of nine measurements.

\subsubsection{Morphology}

The morphology of Lf-GMP nanohydrogels coated with chitosan were evaluated by transmission electron microscopy (TEM) (EM 902A, ZEISS, Germany). TEM samples were prepared by depositing the same suspensions on a carbon-coated copper grid. Before being analysed, samples were air-dried.

\subsubsection{Fourier transform infrared (FTIR) spectroscopy}

In order to confirm the presence of the chitosan in Lf-GMP nanohydrogels, FTIR analyses were carried out with a Thermo Nicolet 6700 Fourier transform infrared spectrometer from Thermo-Fisher Scientific, scanning from 500 to $4000 \mathrm{~cm}^{-1}, 32$ scans were collected for each sample. 


\subsubsection{Circular dichroism (CD)}

The secondary structures of the Lf-GMP nanohydrogels and the effect of chitosan coating was evaluated by circular dichroism. CD spectra were obtained with a Jasco J-810 spectropolarimeter (Jasco Corporation, Japan) equipped with a Peltier temperature controller (PFD $425 \mathrm{~S}$, Jasco, Japan) coupled with a thermostatic bath (AWC 100, Julabo, Germany). The spectra was obtained at $25{ }^{\circ} \mathrm{C}$ using a $10 \mathrm{~mm}$ quartz cuvette (Hellma Analytics, Germany) at wavelength range of $190 \mathrm{~nm}-260 \mathrm{~nm}$. Deionized water was used as a blank.

\subsection{Release experiments}

\subsubsection{Encapsulation of a model bioactive compound}

A model bioactive compound, caffeine, was encapsulated into the Lf-GMP nanohydrogels and characterized in a previous work Bourbon, Cerqueira, \& Vicente (2016). A maximum encapsulation efficiency of $90.02 \pm 2.10 \%$ was verified when $0.03 \mathrm{mg} \mathrm{mL}^{-1}$ was encapsulated in Lf-GMP nanohydrogels. In order to evaluate the effect of chitosan coating on release mechanisms of a model bioactive compound from Lf-GMP nanohydrogels coated with chitosan, caffeine was encapsulated in this system. The encapsulation of caffeine was performed as reported in Bourbon et al. (2016).

Briefly, caffeine solution $\left(0.03 \mathrm{mg} \mathrm{mL}^{-1}\right)$ was added to the mixture solution of Lf $(1.25 \mu \mathrm{M}, \mathrm{pH} 5.0))$ and GMP $(8.3 \mu \mathrm{M}, \mathrm{pH}$ 5.0) with a molar ratio of $1: 7$ of Lf to GMP at $25^{\circ} \mathrm{C}$. After gentle stirring for $30 \mathrm{~min}$, the mixture of Lf-GMP with caffeine was subsequently heated at $80{ }^{\circ} \mathrm{C}$ for $20 \mathrm{~min}$ in a water bath (closed system) to obtain a homogeneously dispersed nanohydrogel solution.

The unbound caffeine was determined after separating the nanohydrogels with encapsulated caffeine from the solution with free caffeine. The separation was performed using an Amicon ${ }^{\mathbb{B}}$ Ultra-0.5 centrifugal filter device with a molecular cut-off $3 \mathrm{kDa}$. The solution of encapsulated caffeine in Lf-GMP nanohydrogels was gently mixed with a chitosan solution $\left(1 \mathrm{mg} \mathrm{mL}^{-1}\right)$ until a VR of 10 of nanohydrogels to chitosan.

\subsubsection{Determination of caffeine release profile}

Release profiles of caffeine were obtained as explained in Bourbon et al. (2016). Briefly, nanohydrogels with caffeine encapsulated $(5 \mathrm{~mL})$ was added into a dialysis membrane with a molecular weight cut-off $8 \mathrm{kDa}$ that was subsequently placed into $40 \mathrm{~mL}$ of $\mathrm{KCl}-\mathrm{HCl}$ buffer solution with $\mathrm{pH} \mathrm{2}$, under magnetic stirring. At appropriate time intervals, $0.25 \mathrm{~mL}$ of supernatant were taken and $0.25 \mathrm{~mL}$ of fresh buffer were added to keep the volume of the release medium constant. The amount of caffeine released was evaluated by measuring the absorbance at $272 \mathrm{~nm}$, respectively (absorbance peak) (Elisa Biotech Synergy HT, Biotek, USA). All release tests were run at least in triplicate.

2.5.2.1. Mathematical modelling. Different release mechanisms that govern compounds release from bio-polymeric systems when immersed in liquid media can be observed (Pinheiro et al., 2013). These mechanisms associated with compounds release can be generally classified in different types: (i) Fickian diffusion, which is related to Brownian motion of molecules (the governing factor for transport mechanism is exclusively a concentration gradient); (ii) Case-II transport, which is driven by the relaxation of the polymer and iii) anomalous transport, a coupling of diffusion and polymeric relaxation (Pinheiro et al., 2013). In order to evaluate the effect of chitosan coating on release properties of Lf-GMP nanohydrogels at acid conditions $(\mathrm{pH} 2)$ to simulate the stomach at $37^{\circ} \mathrm{C}$, the Linear Superposition Model (LSM) was fitted to experimental data
(Berens \& Hopfenberg, 1978). Furthermore, the results of caffeine encapsulated in Lf-GMP nanohydrogels were also fitted with this model in a previous work (Bourbon et al., 2016).

The linear s model for caffeine release from Lf-GMP nanohydrogels can be described by:

$\frac{\mathbf{M}_{\mathbf{t}}}{\mathbf{M}_{\infty}}=\mathbf{X}\left[1-\frac{6}{\pi^{2}} \mathbf{e x p}\left(-\mathbf{k}_{\mathbf{F}} \mathbf{t}\right)\right]+(1-\mathbf{X})\left[1-\exp \left(-\mathbf{k}_{\mathbf{R}} \mathbf{t}\right)\right]$

where $M_{t}$ is the total mass released from the polymeric structure, $k_{F}$ is the Fickian diffusion rate constant, $k_{R_{i}}$ are the relaxation $i$ th rate constants and for most cases, there is only one main polymer relaxation that influences transport and thus the above equation can be simplified using $i=1$ and $X$ is the fraction of compound released by Fickian transport.

2.5.2.2. Non-linear regression analysis. Equation (1) was fitted to data by non-linear regression analysis, using a package of STA-

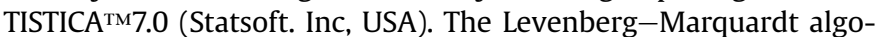
rithm for the least squares function minimization was used. The quality of the regressions was evaluated on the basis of the determination coefficient, $R^{2}$, the squared root mean square error, RMSE (i.e., the square root of the sum of the squared residues (SSE) divided by the regression degrees of freedom) and residuals visual inspection for randomness and normality. $R^{2}$ and SSE were obtained directly from the software. The precision of the estimated parameters was evaluated by the Standardized Halved Width (SHW \%), which was defined as the ratio between the 95\% Standard Error (obtained from the software) and the value of the estimate.

\subsection{Evaluation of in vitro digestibility}

A dynamic in vitro system that simulated the digestive process was used to evaluate the digestibility of Lf-GMP nanohydrogels. Each compartment consists in two connected glass reactors with a flexible wall inside and water is pumped around the flexible walls to maintain the temperature at $37{ }^{\circ} \mathrm{C}$ and to enable the simulation of the peristaltic movements (by the alternate compression and relaxation of the flexible walls). The changes in water pressure are achieved by peristaltic pumps which alter the flow direction according to the time controlling devices connected to them. In vitro gastric digestion was performed as described by other authors (Reis et al., 2008). A volume of $60 \mathrm{~mL}$ of Lf-GMP nanohydrogels (with and without chitosan coating) was introduced into the dynamic gastrointestinal system (gastric compartment) and the experiment was run for a total of $2 \mathrm{~h}$, simulating average physiological conditions of GI tract by the continuous addition of gastric secretions. The gastric secretion consisted of pepsin and lipase in a gastric electrolyte solution $\left(\mathrm{NaCl} 4.8 \mathrm{~g} \mathrm{~L}^{-1}, \mathrm{KCl} 2.2 \mathrm{~g} \mathrm{~L}^{-1}, \mathrm{CaCl}_{2} 0.22 \mathrm{~g} \mathrm{~L}^{-1}\right.$ and $\mathrm{NaHCO}_{3}$ $1.5 \mathrm{~g} \mathrm{~L}^{-1}$ ), secreted at a flow rate of $0.33 \mathrm{~mL} \mathrm{~min}^{-1}$. The $\mathrm{pH}$ was controlled to follow a predetermined curve (Table 1 ) by secreting $\mathrm{HCl}\left(1 \mathrm{~mol} \mathrm{~L}^{-1}\right)$ (Marteau et al., 1991). The temperature was kept at $37{ }^{\circ} \mathrm{C}$ during the whole experiment. Samples were taken at appropriate time intervals during the $2 \mathrm{~h}$ of gastric digestion and keep it at $-20^{\circ} \mathrm{C}$ until analyze. The digestibility assays were conducted in duplicate.

Table 1

Predetermined $\mathrm{pH}$ values in the stomach.

\begin{tabular}{llllllll}
\hline Time (min) & 0 & 5 & 20 & 40 & 60 & 90 & 120 \\
$\mathrm{pH}$ & 4.8 & 4.5 & 4.2 & 2.8 & 2.1 & 1.8 & 1.7 \\
\hline
\end{tabular}




\subsubsection{Native polyacrylamide gel electrophoresis}

In order to evaluate the integrity of nanohydrogel during the digestion experiments, a native-PAGE or "nondenaturing" gel electrophoresis, was performed. Native-PAGE analyses were carried out using the Mini-Protean II dual slab cell system equipped with a PAC 300 power supply (Bio-Rad Laboratories, Hercules, CA, USA). The resolving and stacking gel contained 12.5 and 3.5\% of polyacrylamide, respectively. The gels were stained with silver nitrate methodology (Chevallet, Luche, \& Rabilloud, 2006). Standard marker proteins PageRuler Unstained Broad Range Protein Ladder from Thermo Scientific was used to identify samples by their molecular weight.

\subsubsection{Quantification of $L f$ and GMP}

The concentration of Lf and GMP during gastric digestion was evaluated using high performance liquid chromatography (HPLC). Protein concentrations were assayed by reversed-phase HPLC on a ACE $5 \mathrm{C}_{18}$ column $(250 \times 4.6 \mathrm{~mm}, 5 \mu \mathrm{m}, 300 \AA$, Advanced Chromatography Technologies, Scotland) with an ACE $5 \mathrm{C}_{18}$ guard cartridge (Advanced Chromatography Technologies, Scotland). RPHPLC analysis was performed using an Agilent 1220 HPLC system (Agilent Technologies, Germany). Data acquisition was provided through the Agilent ChemStation software (revision A.10.02) (Agilent Technologies, Palo Alto, CA, USA) and absorbance was measured at the wavelength of $220 \mathrm{~nm}$. A constant flow rate of $0.7 \mathrm{~mL} \mathrm{m^{-1 }}$ was used and the injection volume was $20 \mu \mathrm{L}$. Regarding solvents, solvent A was $0.1 \%(\mathrm{v} / \mathrm{v})$ tri-fluoroacetic (TFA) in Milli-Q water, and solvent B was $0.1 \%(\mathrm{v} / \mathrm{v})$ TFA in $100 \%(\mathrm{v} / \mathrm{v})$ acetonitrile.

Elution was performed as follows: $100 \% \mathrm{~A}$ for $5 \mathrm{~min}$, linear gradient of $0-50 \%$ B for $50 \mathrm{~min}$, from 50 to $100 \%$ B over the next $2.5 \mathrm{~min}$ and then maintained at $100 \%$ B for $2.5 \mathrm{~min}$. Finally, solvent A was increased to $100 \%$ in $5 \mathrm{~min}$ and the column was reequilibrated for 5 min more.

\section{Results and discussion}

\subsection{Characterization of Lf-GMP nanohydrogels coated with chitosan}

\subsubsection{Optimization of chitosan coating}

Since LbL deposition is governed by electrostatic interactions and in order to guarantee a correct deposition of chitosan coating on Lf-GMP nanohydrogels, it is necessary to assure that this polyelectrolyte and nanohydrogels exhibit an opposite charge. Chitosan polysaccharide is a weak polyelectrolyte which their dissociation degree depends strongly on the solution's $\mathrm{pH}$. The chitosan solution was found to have a maximum positive charge $(60.5 \pm 4.2 \mathrm{mV})$ at $\mathrm{pH} 3$ and the Lf-GMP nanohydrogels solution exhibited a maximum negative charge $(-17.11 \pm 1.45 \mathrm{mV})$ at $\mathrm{pH} 5.0(\mathrm{pH}$ that nanohydrogels are prepared) (Bourbon et al., 2015).

In order to guarantee the total deposition of chitosan coating on Lf-GMP nanohydrogels with a minimal impact on nanohydrogels size and polydispersity index, different volume ratios of Lf-GMP nanohydrogels $\left(0.2 \mathrm{mg} \mathrm{mL}^{-1}\right.$ at $\left.\mathrm{pH} 5.0\right)$ to chitosan $\left(1 \mathrm{mg} \mathrm{mL}^{-1}\right.$ at pH 3) were tested (Fig. 1).

The alteration of $\zeta$-potential value is an indicative that the polyelectrolyte is deposited in Lf-GMP nanohydrogels. Fig. 1 shows that increasing the volume of chitosan on the final mixture solution of chitosan and Lf-GMP nanohydrogels increase the $\zeta$-potential, until a point $(V R=5)$ that a constant $\zeta$-potential value $(+56 \pm 4.4 \mathrm{mV})$ is obtained, indicating that the chitosan added fully covered the surface of nanohydrogels. An identical profile was observed by Tan, Wang, and Tam (2008) which applied different layers on methacrylic acid ethyl acrylate nanogels (Tan

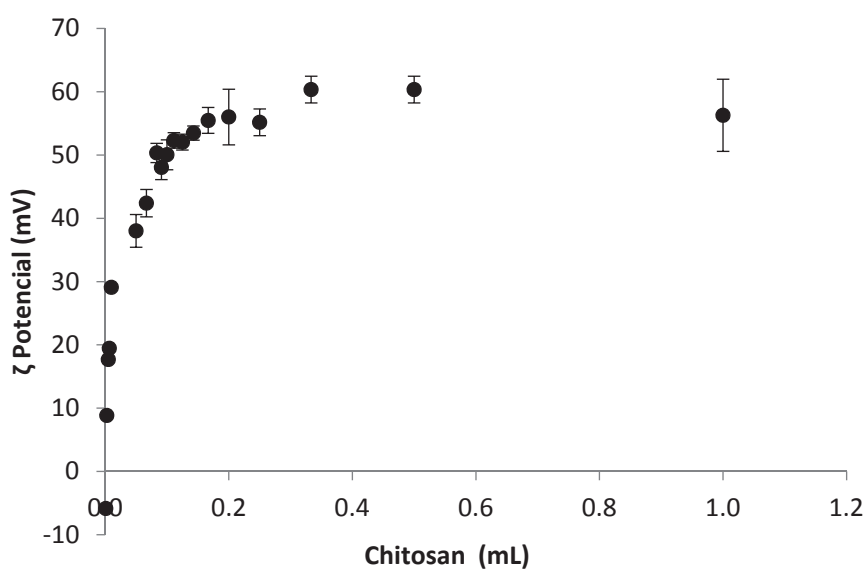

Fig. 1. $\zeta$-potential of Lf-GMP nanohydrogels solution $\left(0.2 \mathrm{mg} \mathrm{mL}^{-1}\right.$, at $\left.\mathrm{pH} 5.0\right)$ coated with chitosan layer ( $\left.1 \mathrm{mg} \mathrm{mL}^{-1}, \mathrm{pH} 3.0\right)$.

et al., 2008).

The optimum amount of chitosan added to Lf-GMP nanohydrogels was determined based on the $\zeta$-potential value and also on the minimum size and PdI of the coated nanohydrogels.

Particle size and PdI curves for the chitosan coating plotted

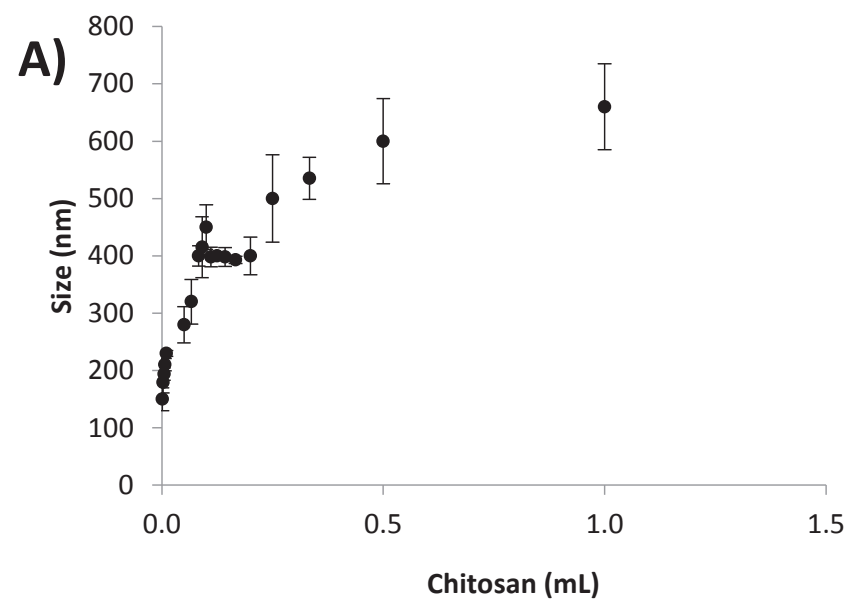

B)

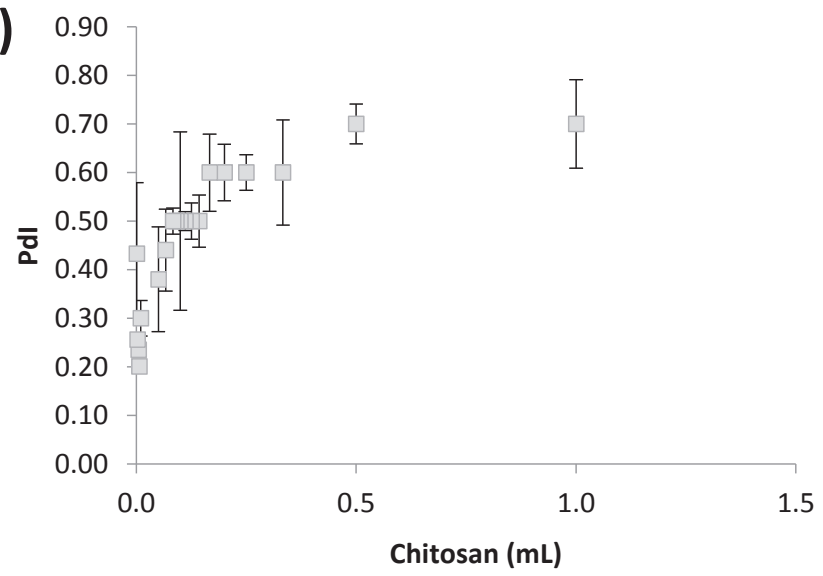

Fig. 2. Size (A) and PdI (B) of Lf-GMP nanohydrogels solution $\left(0.2 \mathrm{mg} \mathrm{mL}^{-1}\right.$, at $\left.\mathrm{pH} 5.0\right)$ coated with chitosan layer (1 $\left.\mathrm{mg} \mathrm{mL}^{-1}, \mathrm{pH} 3.0\right)$. 
against ratio of the volume of chitosan to the volume of Lf-GMP nanohydrogels are shown in Fig. 2a and b) respectively. The application of chitosan coating on Lf-GMP nanohydrogels have influence on the morphology of these nanostructures. It is possible to observe that when VR is higher than 5 , the size and PdI of nanohydrogels increase, suggesting that the addition of more chitosan molecules to the nanohydrogels solution leads to a formation of aggregates (size values $>300 \mathrm{~nm}$ ) and to a heterogeneous solution (PdI values $>0.5$ ). Although, when the VR is lower than 5 , it is possible to observe lower sizes and PdI values of LfGMP nanohydrogels, however the instability of nanohydrogels is higher (lower values of $\zeta$-potential). Therefore, in order to guarantee the use of stable nanohydrogels coated with chitosan and minimum sizes and PdI values, the optimal VR of Lf-GMP nanohydrogels to chitosan is 10 . At this condition, the size of nanohydrogels presents $230 \pm 12 \mathrm{~nm}$, a PdI of $0.22 \pm 0.02$ and a $\zeta_{-}$ potential of $30.0 \pm 0.15 \mathrm{mV}$. According to general colloid chemistry principles, a dispersed system typically loses stability when the magnitude of the $\zeta$-potential is lower than approximately $30 \mathrm{mV}$ (Goetz, 2010).

\subsubsection{FTIR measurements}

In order to confirm the presence of chitosan in Lf-GMP nanohydrogels, FTIR spectra of the Lf-GMP nanohydrogels and Lf-GMP nanohydrogels coated with chitosan can be seen in Fig. 3.

FTIR analyses were used to evaluate the interactions between LfGMP nanohydrogels and the chitosan applied as a coating. The FTIR spectroscopy spectra of chitosan depict characteristic absorption bands at 3352 and $2932 \mathrm{~cm}^{-1}$, which represent the $-\mathrm{OH}$ and $-\mathrm{CH}_{2}$ aliphatic groups, and bands at 1563 and $1414 \mathrm{~cm}^{-1}$, which represent the $\mathrm{NH}$-group bending vibration and vibrations of $-\mathrm{OH}$ group of the primary alcoholic group, respectively (Kumar \& Koh, 2012). The amino group has a characteristic absorption band in the region of $3400-3500 \mathrm{~cm}^{-1}$, which is masked by the broad absorption band from the $-\mathrm{OH}$ group. The shoulders at $1635 \mathrm{~cm}^{-1}$ represents the $\mathrm{C}=\mathrm{O}$ groups and suggests chitosan is a partially deacetylated product (Sionkowska, Wisniewski, Skopinska, Kennedy, \& Wess, 2004).

The interactions between chitosan and proteins are represented by amide I band (between 1600 and $1700 \mathrm{~cm}^{-1}$ ), amide II (around $1536 \mathrm{~cm}^{-1}$ ) and by NH and CO deformations (1580-1490 $\mathrm{cm}^{-1}$ and $1700-1630 \mathrm{~cm}^{-1}$ range, respectively) (Pranoto, Rakshit, \& Salokhe, 2005; Silva et al., 2007). It is possible observe a shift of $\mathrm{NH}$ and $\mathrm{CO}$ deformation bands from 1584 to $1536 \mathrm{~cm}^{-1}$ and from 1650 to

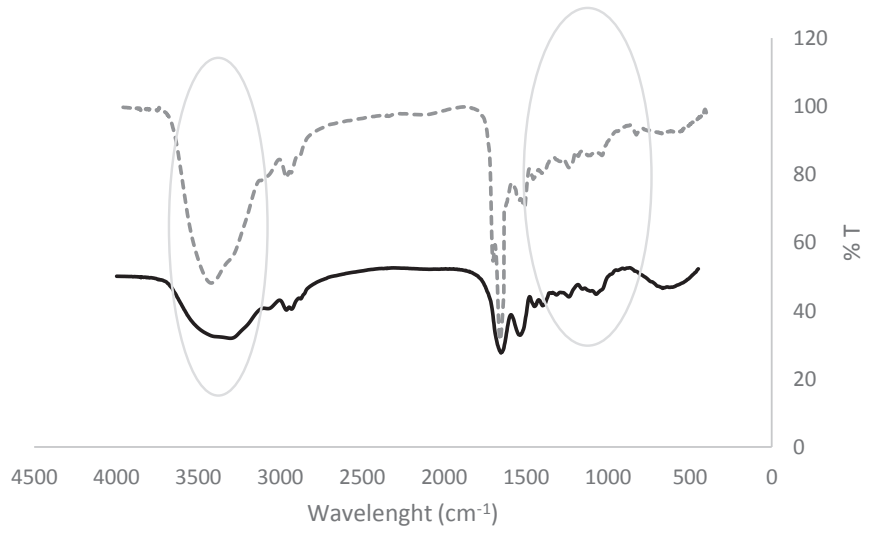

Fig. 3. FTIR spectra of Lf-GMP nanohydrogels (-), Lf-GMP nanohydrogels with chitosan coating (VR $=10$ for Lf-GMP nanohydrogels: chitosan) (-).

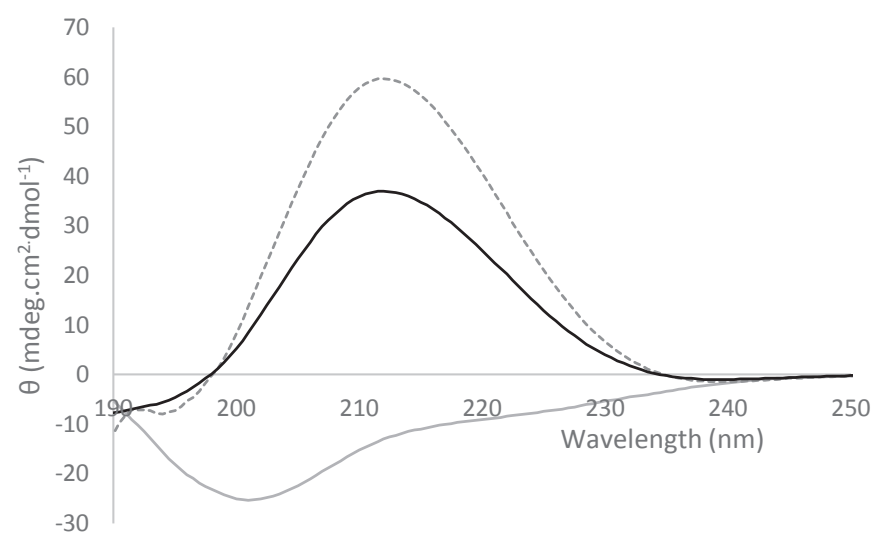

Fig. 4. CD spectra of Lf-GMP nanohydrogels (-), chitosan (---) and Lf-GMP nanohydrogels with chitosan coating (VR $=10$ for Lf-GMP nanohydrogels: chitosan) (-).

$1630 \mathrm{~cm}^{-1}$ (Cai et al., 2010). These shifts suggest a specific chemical interaction occurring between functional groups of protein-based nanohydrogels and active groups of the chitosan (highlighted in Fig. 3).

\subsubsection{Circular dichroism}

CD spectra of Lf-GMP nanohydrogels, Lf-GMP nanohydrogels with chitosan coating and chitosan alone are shown in Fig. 4.

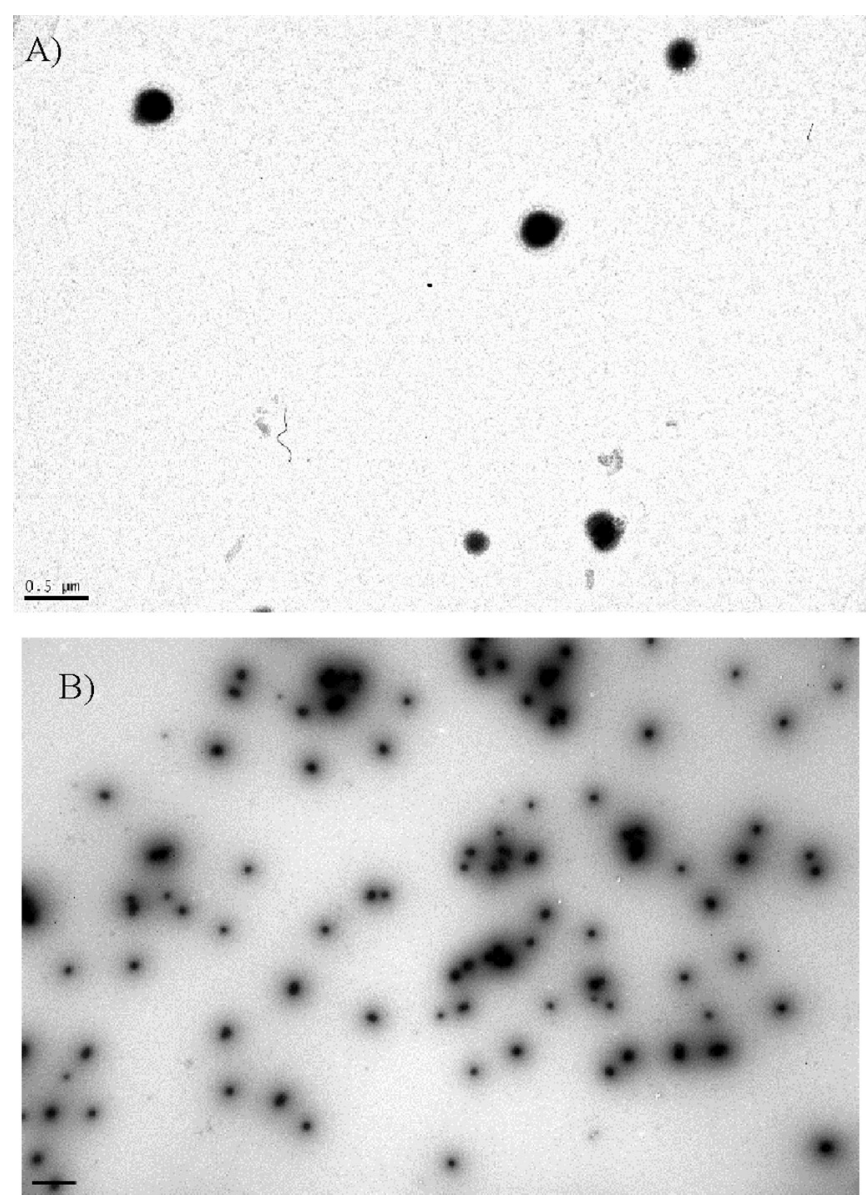

Fig. 5. TEM images of: A) Lf-GMP nanohydrogels and B) Lf-GMP nanohydrogels with chitosan coating. The scale bar is (A). $0.5 \mu \mathrm{m}$ and (B) $2 \mu \mathrm{m}$. 


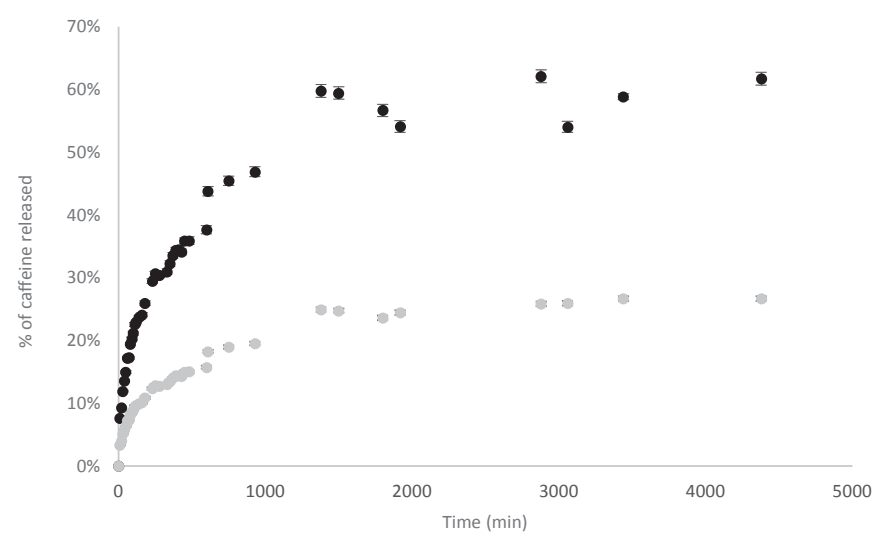

Fig. 6. Release profile of caffeine: through Lf-GMP nanohydrogels ( $)$ and through LfGMP nanohydrogels with chitosan coating (॰), at $\mathrm{pH} 2,37^{\circ} \mathrm{C}$.
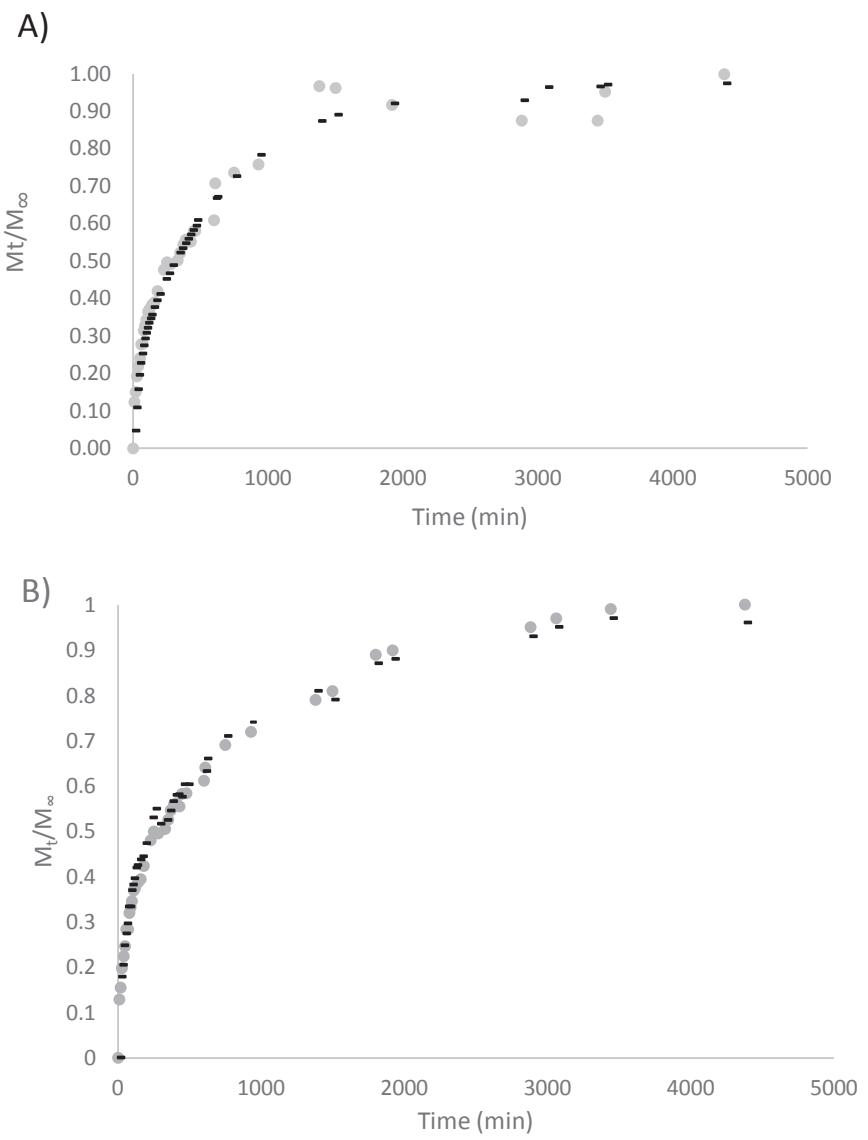

Fig. 7. Linear Superposition Model (LSM) description of caffeine release from: a) LfGMP nanohydrogels and b) Lf-GMP nanohydrogels with chitosan coating, at $\mathrm{pH} 2$ and at $37{ }^{\circ} \mathrm{C}$ (experimental results $(\bullet)$; model-generated values $(-$ ).

CD spectroscopy has been used to characterize the interactions between molecules, namely between proteins, once this technique allows evaluate the secondary structures of proteins and polypeptides in solution (Greenfield, 1996). The evaluation of interaction between chitosan and proteins are scarce. In fact, the CD signals induced from chitosan are superposed with each other in some cases and this causes the spectrum to be poorly resolved and makes it difficult to analyse structures (Singh \& Dutta, 2009).

From Fig. 4 it is possible to observe that the interaction of chitosan with Lf-GMP nanohydrogels did not show any relevant information of the effect of chitosan on secondary structure of protein nanohydrogels. CD spectra of Lf-GMP nanohydrogels shows the presence of $\beta$-sheet structure (negative band around $217 \mathrm{~nm}$ ) and a presence of unordered conformation that is observed by a single band near $200 \mathrm{~nm}$. When a chitosan coating is applied on Lf-GMP nanohydrogels, it is possible to observe that the negative bands are not visible (typical of secondary structure of proteins) and positive band appears, corresponding to chitosan conformation. The $\mathrm{CD}$ spectra obtained for chitosan are in accordance with the spectra reported by Wu et al. (2005). These authors reported that the chitosan $C D$ spectra with strong absorbance is due to the chiral system (Wu et al., 2005). Comparing the chitosan alone with chitosan in Lf-GMP nanohydrogels CD spectra it is possible to observe a decrease of band intensity and a small shift from 209 (chitosan) to $212 \mathrm{~nm}$ (Lf-GMP nanohydrogels coated with chitosan). These differences can suggest that an interaction was established during structure assembly (Diniz et al., 2014).

\subsubsection{Morphology}

Morphological characteristics of Lf-GMP nanohydrogels coated with chitosan layer and Lf-GMP nanohydrogels were examined using TEM (Fig. 5).

Microscopy images of the Lf-GMP nanohydrogels show that the particles are spherical and present an average diameter around $170 \mathrm{~nm}$, which is in accordance with previous results reported in Bourbon et al. (2015). After the deposition of chitosan coating, it is possible to observe that nanohydrogels maintains the spherical shape and the size value is around $200 \mathrm{~nm}$. This value was confirmed by Dynamic Light Scattering measurements that reveal a mean size of $230 \pm 12 \mathrm{~nm}$ and PdI of $0.22 \pm 0.02$. The difference between the size values obtained by TEM and DLS can be related with the drying process of nanohydrogels used to prepare the samples for TEM visualization. The low PdI value is corroborated by TEM image, which demonstrated an Lf-GMP nanohydrogels solution with low presence of aggregates.

\subsubsection{In vitro release}

Following a detailed physical characterization of Lf-GMP nanohydrogels with chitosan coating, the capability of coated nanohydrogels in interfere with release mechanisms of active compound was evaluated. The influence of chitosan coating on the release profile of caffeine from Lf-GMP nanohydrogels can be observed in Fig. 6.

The knowledge of the effect of the chitosan on the mass released can be of greatest importance for the application of

Table 2

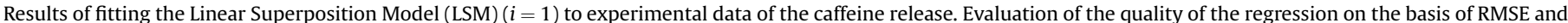
$R^{2}$. Estimates precision is evaluated using the SHW \% (in parenthesis).

\begin{tabular}{|c|c|c|c|c|c|}
\hline System & RMSE & $R^{2}$ & $X$ & $k_{F}\left(\min ^{-1}\right)$ & $K_{R}\left(\min ^{-1}\right)$ \\
\hline Lf-GMP nanohydrogel & 0.160 & 0.903 & $0.410(48.20 \%)$ & $0.090(26.56 \%)$ & $0.001(54.76 \%)$ \\
\hline Lf-GMP nanohydrogels with chitosan coating & 0.089 & 0.987 & $0.314(32.78 \%)$ & $0.261(32.65 \%)$ & $0.101(23.24 \%)$ \\
\hline
\end{tabular}




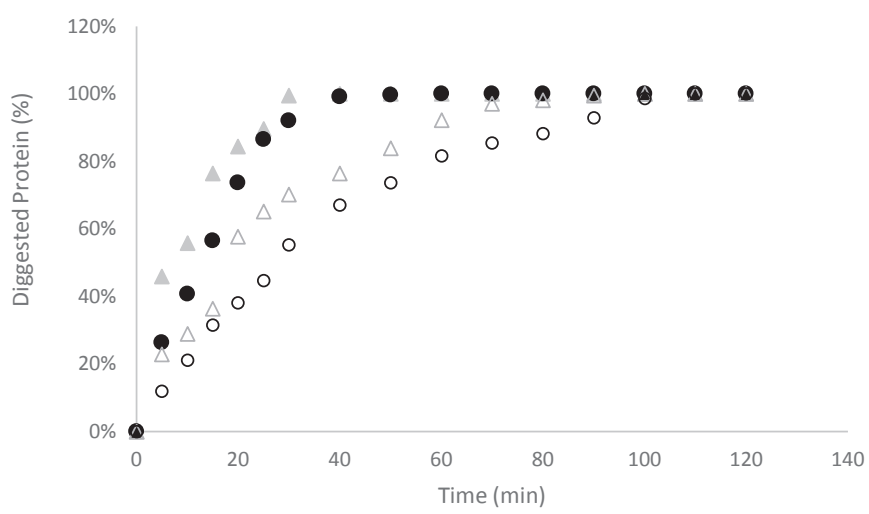

Fig. 8. Gastric digestion kinetic of Lf-GMP nanohydrogels (Lf ( $\Delta$ ) and GMP ( $)$ - full symbols) and Lf-GMP nanohydrogels coated with chitosan (Lf $(\triangle)$ and GMP $(O)$ - open symbols).

these nanostructures on food products. Furthermore, once applied on a food system, the release of bioactive compound can occur after or before the ingestion. Therefore, the release behavior of caffeine from Lf-GMP nanohydrogels with chitosan coating was evaluated at $37^{\circ} \mathrm{C}$, which is the temperature within human body and at $\mathrm{pH} 2$ which is the $\mathrm{pH}$ correspondent to the stomach.

The deposition of a coating in Lf-GMP nanohydrogels revealed the ability to modify the caffeine release through nanohydrogels matrix. Fig. 6 shows that the amount of caffeine released (27\%) from Lf-GMP nanohydrogels with chitosan coating was lower when compared with nanohydrogel without coating (62\%). Also, it is possible to observe that the caffeine released during the initial times (burst release) became gentler when the chitosan coating was present in Lf-GMP nanohydrogels. This behavior can be due to the fact that with the coating, the caffeine molecules move slowly through the matrix, therefore mitigating the burst release phenomenon. Tan et al. (2008) reported a similar behavior when evaluated the release profile of procaine hydrochloride in nanogels with layers.

In order to explore the effect of chitosan on release mechanisms of bioactive compound, the LSM model (Equation (1)) was fitted to experimental data (Fig. 7).

Fig. 7 and Table 2 shows that the experimental data were adequately fitted to LSM (Equation (1)). The good regression quality $\left(R^{2}>0.90\right)$ and the good precision $(S H W \%<55)$ of estimated parameters shows that this model adequately describes the release mechanisms involved on caffeine release. Suggesting that the caffeine released through Lf-GMP nanohydrogels and through LfGMP nanohydrogels with chitosan coating is governed by the combination of Fick's diffusion and the relaxation of polymer, with only one main relaxation of the matrix.

Results also show that with application of chitosan layer, the $X$ value (which is defined as $\frac{M_{\infty, F}}{M_{\mathrm{t}}}$ ) is lower than 0.5 , indicating that relaxation mechanism is the main phenomena on caffeine release. When compared with nanohydrogels without coating, the $X$ value is lower, suggesting that the relaxation process is more accentuated. In fact, FTIR and CD measurements suggested that the interaction of chitosan with protein nanohydrogels promoted structural and chemical changes, contributing for different properties of matrix.

A)

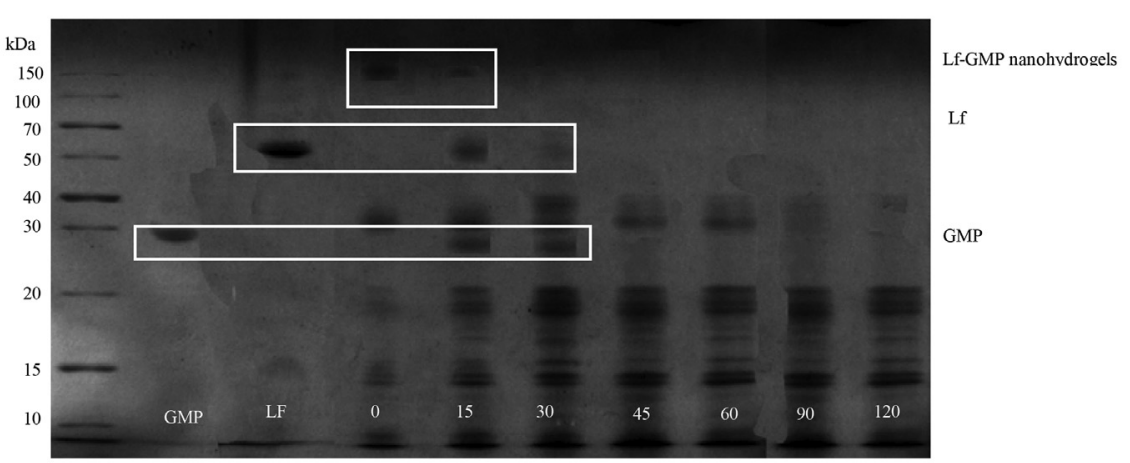

B)

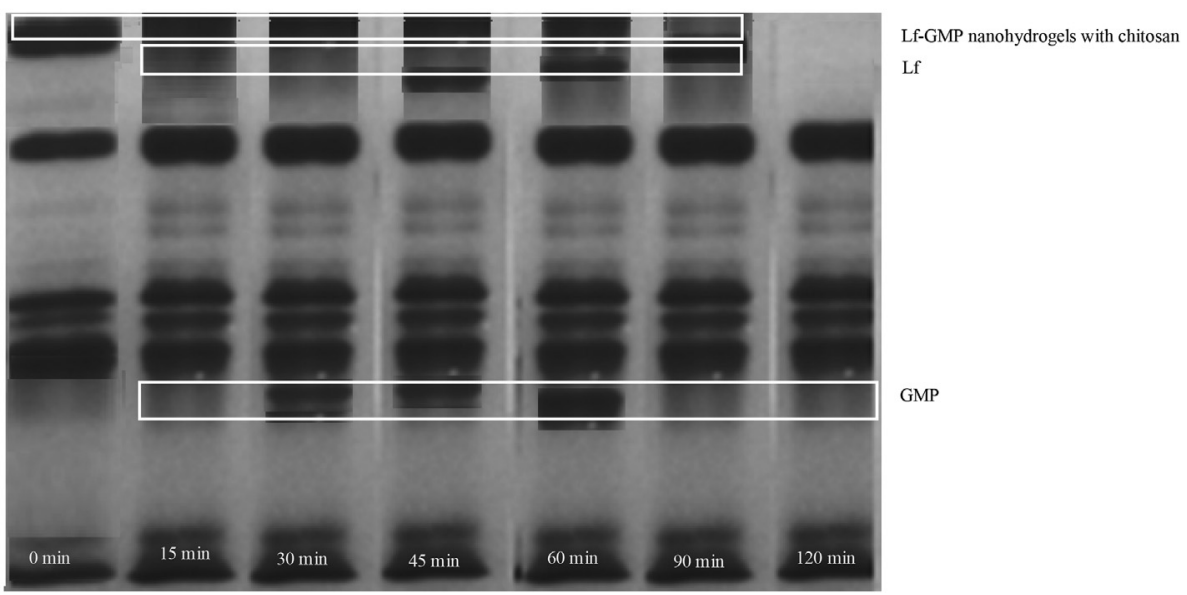

Fig. 9. Native Electrophorese of gastric kinetic digestion of: A) Lf-GMP nanohydrogels and B) Lf-GMP nanohydrogels with chitosan coating. 

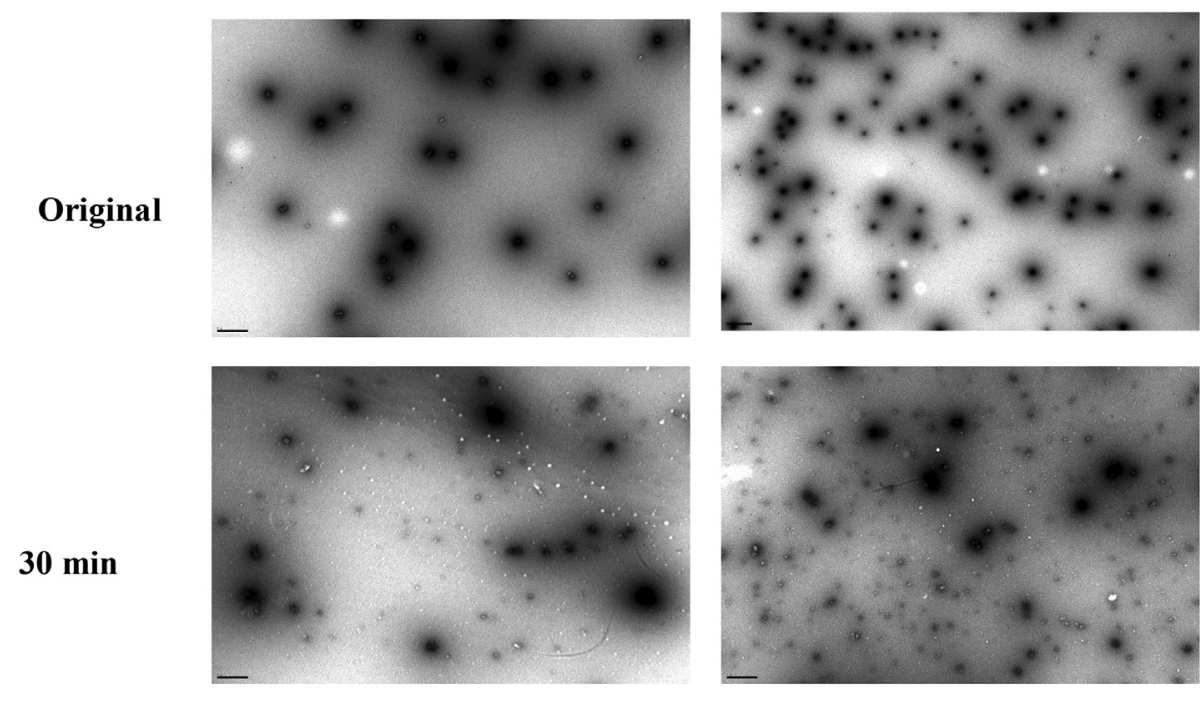

$60 \mathrm{~min}$
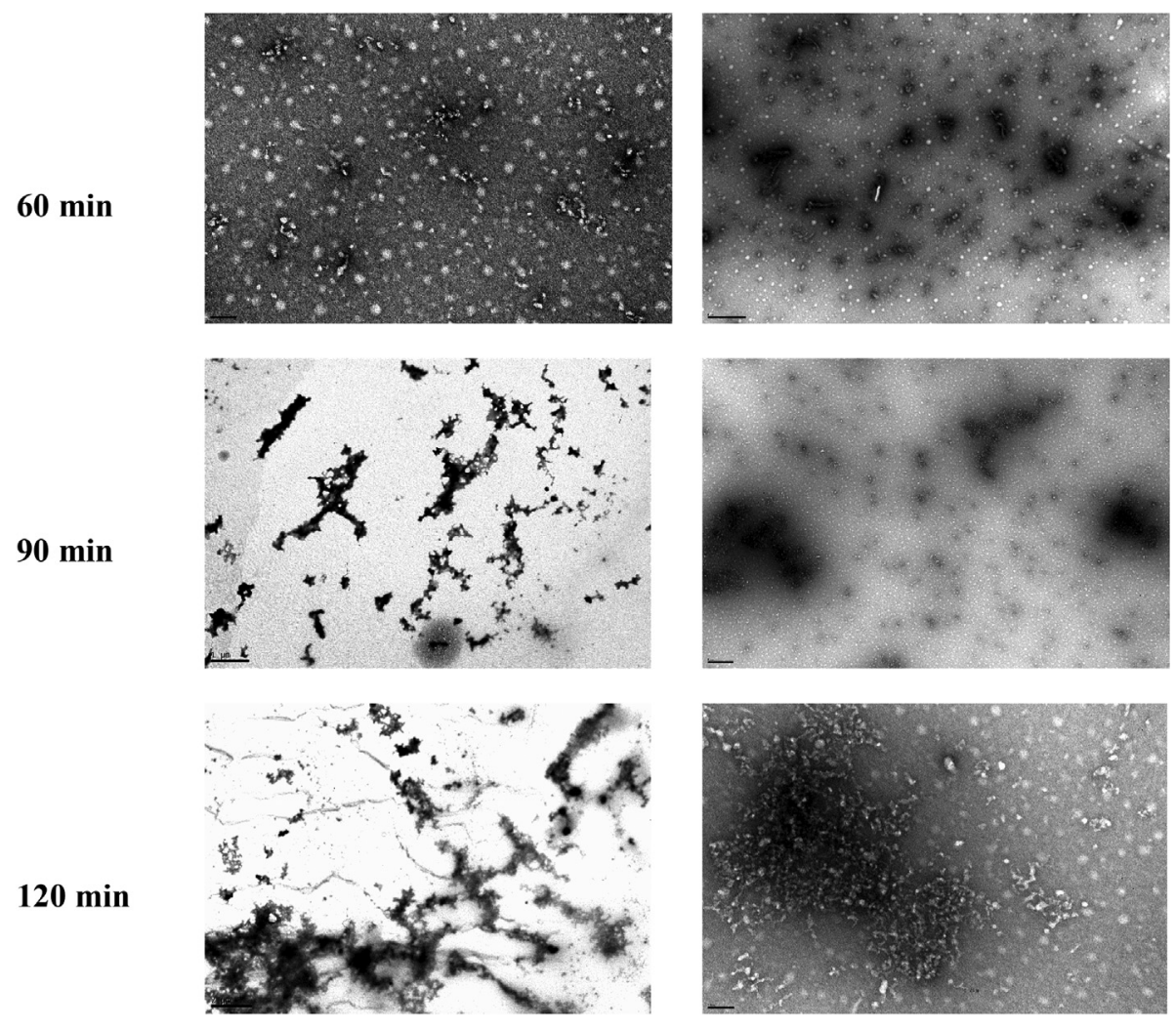

A
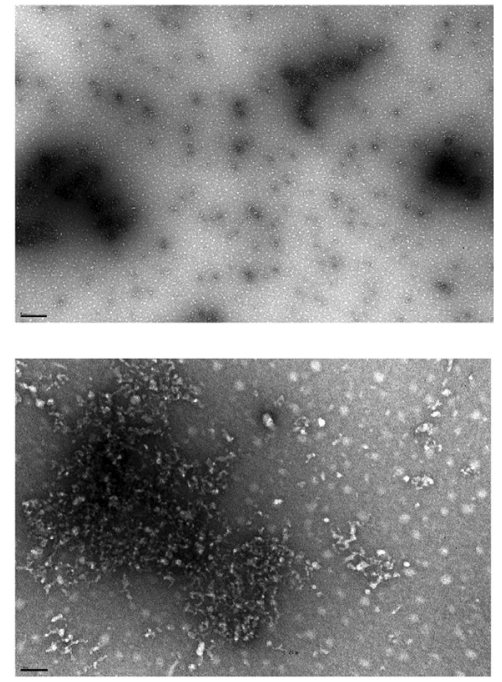

B

Fig. 10. TEM images of gastric digestion of Lf-GMP nanohydrogels (A) and Lf-GMP nanohydrogels with chitosan coating (B) as a function of time.

It was also observed that the Fickian rate constant $\left(K_{F}\right)$ increase in Lf-GMP nanohydrogels with chitosan coating, revealing that caffeine molecules were released faster during the Fick's diffusion than in Lf-GMP nanohydrogels without coating. As for the relaxation component of transport, relaxation rate constant $\left(K_{R}\right)$, also increase in nanohydrogels with chitosan coating. This should be expected since this is a property of polymer (Vrentas, Jarzebski, \& Duda, 1975).

\subsection{Gastric digestion of $L f$-GMP nanohydrogels}

In order to evaluate the stability of Lf-GMP nanohydrogels during gastric digestion, these structures were submitted to gastric environmental ( $\mathrm{pH}$, enzymes, ionic strength, and controlled temperature) with the simulated peristaltic movements in a dynamic digestion system. The influence of chitosan coating on degradation of Lf-GMP nanohydrogels was evaluated by the quantification of proteins (Lf and GMP) during the gastric digestion (Fig. 8).

During the gastric digestion, different parameters could have a high influence on protein structures. The effect of $\mathrm{pH}$ and ionic strength are two examples of important parameters on proteins properties that are extremely well reported in literature (Chen, Remondetto, \& Subirade, 2006; Davidov-Pardo, Joye, \& 
McClements, 2015; Gunasekaran, Ko, \& Xiao, 2007; Law \& Leaver, 2000). In stomach the $\mathrm{pH}$ value is gradually decreasing and the ionic strength increasing with the addition of gastric fluids. Accomplished to this conditions, there are a presence of enzymes, in particular the gastric pepsin, which is responsible for the protein hydrolyse.

Fig. 8 shows that during the gastric digestion the proteins are completely hydrolysed. It is notorious that these process is more evident during the initial time of digestion, as is observed by the higher percentages of protein degradation at initial time. Increasing the digestion time it was also observed an increase of the presence of small peptides, which resulted from protein hydrolysis that occurs during digestion (results not shown).

This behaviour is in accordance with reported by Guo, Ye, Lad, Dalgleish, and Singh (2014), who analysed the digestion of whey proteins under simulated gastrointestinal conditions and observed that during the first $30 \mathrm{~min}$ the proteins were digested slightly faster and the presence of small peptides increased.

The application of a chitosan coating revealed to have a high impact on the decrease of protein degradation. This is visible by the presence of the intact proteins during a higher period of time and by the less accentuated slope of protein degradation. In fact, it is possible to observe that around $30 \mathrm{~min}$, all the Lf and GMP were hydrolysed in the system without coating, whereas with chitosan coating the full protein hydrolysis only occurs at the of $90 \mathrm{~min}$ of gastric digestion. Chitosan molecules demonstrated to be able to improve the stability of proteins at gastric conditions. This behaviour can be due to the fact that chitosan has the ability to swell and to form a hydrogel in the acidic gastric juice, protecting protein system (Anal, Bhopatkar, Tokura, Tamura, \& Stevens, 2003).

However, these results only show the degradations of nanohydrogels constituents, which do not mean that the particle was intact during the period of time that the proteins were not fully hydrolysed. In order to evaluate the nanostructure stability, a native electrophorese was performed for both systems (Fig. 9).

Native electrophoreses of Lf-GMP nanohydrogels (Fig. 9A)) shows that a presence of a high molecular weight aggregate is present until 15 min of gastric digestion, suggesting that the LfGMP nanohydrogels structure is almost intact until this period. After that, it is possible to observe the presence of the characteristics bands of Lf and GMP, indicating that these proteins are present in solution but not are part of nanohydrogels structure. These proteins are visible until $30 \mathrm{~min}$ of gastric digestions, which corroborates the results obtained by HPLC measurements.

The native electrophorese of Lf-GMP coated with chitosan (Fig. 9B)) suggests that nanohydrogels are intact until 60 min of gastric digestion. The high molecular weight band characteristic of protein aggregates (nanohydrogels) turns softer, indicating a higher degradation of protein structure and at $90 \mathrm{~min}$, it is possible to observe a soft band that indicates that some of nanohydrogels structures are present however most of nanohydrogels have been hydrolysed. Also, it is possible to observe an increase of presence of Lf and GMP bands with the gastric digestion. At 15 min of gastric digestion, a slight presence of LF and GMP is visible, indicating that a part of nanohydrogels is being degraded, and with increase of time it is possible to observe stronger bands, suggesting the higher present of these free proteins in solution. At $90 \mathrm{~min}$ of digestion it is visible that GMP was almost completely hydrolysed and Lf is still present in solution.

TEM images of gastric digestion of nanohydrogels and nanohydrogels with chitosan coating are shown in Fig. 10. It is possible observe that after $30 \mathrm{~min}$, almost particles of nanohydrogels were destroyed (Fig. $10 \mathrm{~A}$ ) and networks of proteins and enzymes are visible after this period of time. Images of nanohydrogels with chitosan coating during gastric digestion (Fig. 10 B) reveals that spherical particles are present until 90 min and after this time only is visible proteins denatured. These results are in accordance with results obtained by HPLC and electrophorese.

\section{Conclusions}

Chitosan coating was successfully applied in Lf-GMP nanohydrogels, as a strategy to improve the stability of protein systems in gastric conditions. Lf-GMP nanohydrogels with chitosan coating maintained their spherical shape and homogeneous dispersion in solution. It was observed by FTIR and CD that chitosan established chemical interactions with Lf-GMP nanohydrogels. The application of chitosan revealed to have influence on release mechanisms of bioactive compounds from Lf-GMP nanohydrogels. In vitro gastric digestion showed that chitosan improved the stability of proteins in gastric conditions (proteins' hydrolysis were slower) and allow maintain the intact structure for longer periods of gastric digestion. These findings provide a useful guide in the design of coated protein structures for controlled bioactive compounds delivery applications.

\section{Acknowledgements}

Ana I. Bourbon, Ana C. Pinheiro and Miguel A. Cerqueira acknowledge the Fundação para a Ciência e Tecnologia (FCT, Portugal) for their fellowships SFRH/BD/73178/2010, SFRH/BPD/ $101181 / 2014$ and SFRH/BPD/72753/2010, respectively). The authors would like to acknowledge Jorge Padrão from CEB, University of Minho for helping in antimicrobial measurements and to Rui Fernandes from IBMC, University of Porto for assistance in taking the TEM pictures. Also, the authors would like to thank the FCT Strategic Project of UID/BIO/04469/2013 unit, the project RECI/BBB-EBI/ 0179/2012 (FCOMP-01-0124-FEDER-027462) and the project "BioInd - Biotechnology and Bioengineering for improved Industrial and Agro-Food processes", REF. NORTE-07-0124-FEDER-000028 Co-funded by the Programa Operacional Regional do Norte (ON.2 O Novo Norte), QREN, FEDER.

\section{References}

Anal, A. K., Bhopatkar, D. Tokura, S., Tamura, H., \& Stevens, W. F. (2003). Chitosanalginate multilayer beads for gastric passage and controlled intestinal release of protein. Drug Development and Industrial Pharmacy, 29(6), 713-724.

Berens, A. R., \& Hopfenberg, H. B. (1978). Diffusion and relaxation in glassy polymer powders: 2. Separation of diffusion and relaxation parameters. Polymer, 19(5), 489-496.

Boddohi, S., Almodóvar, J., Zhang, H., Johnson, P. A., \& Kipper, M. J. (2010). Layer-bylayer assembly of polysaccharide-based nanostructured surfaces containing polyelectrolyte complex nanoparticles. Colloids and Surfaces B: Biointerfaces, 77(1), 60-68.

Bourbon, A. I. Cerqueira, M. A. \& Vicente, A. A. (2016). Encapsulation and controlled release of bioactive compounds in lactoferrin-glycomacropeptide nanohydrogels: curcumin and caffeine as model compounds. Journal of Food Engineering, 180, 110-119.

Bourbon, A. I., Pinheiro, A. C Carneiro-da-Cunha, M. G Pereira, R. N Cerqueira, M. A., \& Vicente, A. A. (2015). Development and characterization of lactoferrin-GMP nanohydrogels: evaluation of $\mathrm{pH}$, ionic strength and temperature effect. Food Hydrocolloids, 48(0), 292-300.

Cai, J., Yang, J., Wang, C., Hu, Y., Lin, J., \& Fan, L. (2010). Structural characterization and antimicrobial activity of chitosan (CS-40)/nisin complexes. Journal of Applied Polymer Science, 116(6), 3702-3707.

Cerqueira, M. A., Pinheiro, A. C. Silva, H. D., Ramos, P. E., Azevedo, M. A., FloresLópez, M. L., et al. (2014). Design of bio-nanosystems for oral delivery of functional compounds. Food Engineering Reviews, 6(1-2), 1-19.

Chen, L., Remondetto, G. E., \& Subirade, M. (2006). Food protein-based materials as nutraceutical delivery systems. Trends in Food Science \& Technology, 17(5), $272-283$.

Chevallet, M., Luche, S., \& Rabilloud, T. (2006). Silver staining of proteins in 
polyacrylamide gels. Nature Protocols, 1(4), 1852-1858.

Chew, S.-C., Tan, C.-P., Long, K., \& Nyam, K.-L. (2015). In-vitro evaluation of kenaf seed oil in chitosan coated-high methoxyl pectin-alginate microcapsules. Industrial Crops and Products, 76(0), 230-236.

Davidov-Pardo, G., Joye, I. J., \& McClements, D. J. (2015). Chapter nine - food-grade protein-based nanoparticles and microparticles for bioactive delivery: fabrication, characterization, and utilization. In D. Rossen (Ed.), Advances in protein chemistry and structural biology (pp. 293-325). Academic Press.

Decher, G. (2003). Polyelectrolyte multilayers, an overview. KGaA: Wiley-VCH Verlag $\mathrm{GmbH} \& \mathrm{Co}$.

Diniz, R. S., Coimbra, J. S. d. R., Teixeira, Á. V. N. d. C., da Costa, A. R., Santos, I. J. B., Bressan, G. C., et al. (2014). Production, characterization and foamability of $\alpha$ lactalbumin/glycomacropeptide supramolecular structures. Food Research International, 64(0), 157-165.

Donato-Capel, L., Garcia-Rodenas, C. L., Pouteau, E., Lehmann, U., Srichuwong, S., Erkner, A., et al. (2014). Chapter 14-Technological means to modulate food digestion and physiological response. In M. Boland, M. Golding, \& H. Singh (Eds.), Food structures, digestion and health (pp. 389-422). San Diego: Academic Press.

Goetz, D. (2010). Characterization of liquids, nano- and microparticulates, and porous bodies using ultrasound. Elsevier.

Greenfield, N. J. (1996). Methods to estimate the conformation of proteins and polypeptides from circular dichroism data. Analytical Biochemistry, 235(1), 1-10.

Gunasekaran, S., Ko, S., \& Xiao, L. (2007). Use of whey proteins for encapsulation and controlled delivery applications. Journal of Food Engineering, 83(1), 31-40.

Guo, Q., Ye, A., Lad, M., Dalgleish, D., \& Singh, H. (2014). Behaviour of whey protein emulsion gel during oral and gastric digestion: effect of droplet size. Soft Matter, 10(23), 4173-4183.

Hirsjärvi, S., Qiao, Y., Royere, A., Bibette, J., \& Benoit, J.-P. (2010). Layer-by-layer surface modification of lipid nanocapsules. European Journal of Pharmaceutics and Biopharmaceutics, 76(2), 200-207.

Khopade, A. J., \& Caruso, F. (2004). Two-component, ultrathin microcapsules prepared by a core-mediated layer-by-layer approach. Chemistry of Materials, 16(11), 2107-2112.

Kittitheeranun, P., Sajomsang, W., Phanpee, S., Treetong, A., Wutikhun, T., Suktham, K., Puttipipatkhachorn, S., Ruktanonchai, U. R.., Layer-by-Layer Engineered Nanocapsules of curcumin with improved cell activity. International Journal of Pharmaceutics $(0)$.

Kotov, N. A. (2003). Layer-by-layer assembly of nanoparticles and nanocolloids: Intermolecular interactions, structure and materials perspectives. Wiley-VCH Verlag GmbH \& Co. KGaA.

Kumar, S., \& Koh, J. (2012). Physiochemical, circular dichroism-induced helical conformation and optical property of chitosan azo-based amino methanesulfonate complex. Journal of Applied Polymer Science, 124(6), 4897-4903.

Law, A. J. R., \& Leaver, J. (2000). Effect of pH on the thermal denaturation of whey proteins in milk. Journal of Agricultural and Food Chemistry, 48(3), 672-679.

Li, J. K., Wang, N., \& Wu, X. S. (1998). Gelatin nanoencapsulation of protein/peptide drugs using an emulsifier-free emulsion method. Journal of Microencapsulation, 15(2), 163-172.

Marteau, P., Pochart, P., Mahé, S., Crine, L., Huneau, J. F., \& Tomé, D. (1991). Gastric emptying but not orocecal transit time differs between milk and yoghurt in lactose digesters. Gastroenterology, 100, 4535.

Martins, J., Ramos, Ó., Pinheiro, A., Bourbon, A., Silva, H., Rivera, M., et al. (2015). Edible bio-based nanostructures: delivery, absorption and potential toxicity.
Food Engineering Reviews, 1-23.

Nabil, S., Gauthier, S., Drouin, R., Poubelle, P., \& Pouliot, Y (2011). In vitro digestion of proteins and growth factors in a bovine whey protein extract as determined using a computer-controlled dynamic gastrointestinal system (TIM-1). Food Digestion, 2(1-3), 13-22.

Pinheiro, A. C., Bourbon, A. I., Vicente, A. A., \& Quintas, M. A. C. (2013). Transport mechanism of macromolecules on hydrophilic bio-polymeric matrices diffusion of protein-based compounds from chitosan films. Journal of Food Engineering, 116(3), 633-638.

Pranoto, Y., Rakshit, S. K., \& Salokhe, V. M. (2005). Enhancing antimicrobial activity of chitosan films by incorporating garlic oil, potassium sorbate and nisin. LWT Food Science and Technology, 38(8), 859-865.

Rastall, R. A. (2010). Toward second-generation carbohydrate functional food ingredients, nondigestible carbohydrates and digestive health (pp. 223-244). WileyBlackwell.

Reis, P. M., Raab, T. W., Chuat, J. Y., Leser, M. E., Miller, R., Watzke, H. J., et al. (2008) Influence of surfactants on lipase fat digestion in a model gastro-intestinal system. Food Biophysics, 3(4), 370-381.

Sato, K., Yoshida, K., Takahashi, S., \& Anzai, J.-i. (2011). pH- and sugar-sensitive layerby-layer films and microcapsules for drug delivery. Advanced Drug Delivery Reviews, 63(9), 809-821.

Shaji, J., \& Patole, V. (2008). Protein and peptide drug delivery: oral approaches. Indian Journal of Pharmaceutical Sciences, 70(3), 269-277.

Silva, S. S., Goodfellow, B. J., Benesch, J., Rocha, J., Mano, J. F., \& Reis, R. L. (2007) Morphology and miscibility of chitosan/soy protein blended membranes. Carbohydrate Polymers, 70(1), 25-31.

Singh, J., \& Dutta, P. K. (2009). Preparation, circular dichroism induced helical conformation and optical property of chitosan acid salt complexes for biomedical applications. International Journal of Biological Macromolecules, 45(4), 384-392.

Sionkowska, A., Wisniewski, M., Skopinska, J., Kennedy, C. J., \& Wess, T. J. (2004). Molecular interactions in collagen and chitosan blends. Biomaterials, 25(5) 795-801.

Somchue, W., Sermsri, W., Shiowatana, J., \& Siripinyanond, A. (2009). Encapsulation of $\alpha$-tocopherol in protein-based delivery particles. Food Research International 42(8), 909-914.

Tan, J. P. K., Wang, Q., \& Tam, K. C. (2008). Control of burst release from nanogels via layer by layer assembly. Journal of Controlled Release, 128(3), 248-254.

Vrentas, J. S., Jarzebski, C. M., \& Duda, J. L. (1975). A Deborah number for diffusion in polymer-solvent systems. AIChE Journal, 21(5), 894-901.

Wang, R. Tian, Z. \& Chen, L. (2011). Nano-encapsulations liberated from barley protein microparticles for oral delivery of bioactive compounds. International Journal of Pharmaceutics, 406(1-2), 153-162.

Wong, J. E., Müller, C. B., Diez-Pascual, A. M., \& Richtering, W. (2009). Study of layerby-layer films on thermoresponsive nanogels using temperature-controlled dual-focus fluorescence correlation spectroscopy. The Journal of Physical Chemistry B, 113(49), 15907-15913.

Wu, Y., Seo, T., Maeda, S., Sasaki, T., Irie, S., \& Sakurai, K. (2005). Circular dichroism induced by the helical conformations of acylated chitosan derivatives bearing cinnamate chromophores. Journal of Polymer Science Part B: Polymer Physics, 43(11), 1354-1364.

Yvon, M., Beucher, S., Scanff, P., Thirouin, S., \& Pelissier, J. P. (1992). In vitro simulation of gastric digestion of milk proteins: comparison between in vitro and in vivo data. Journal of Agricultural and Food Chemistry, 40(2), 239-244. 